\title{
Restoration of Fingerprints from a Mummified Cadaver
}

\author{
Etsuko Iwakami, Seisaku Uchigasaki and Jian Tie*
}

Division of Legal Medicine, Department of Social Medicine, Nihon University School of Medicine, Tokyo 173-8610, Japan

\begin{abstract}
Fingerprints are widely used as the most reliable means of individual identification in forensic science. However postmortem changes of the skin always make it difficult to obtain fingerprints. To restore fingerprints of mummified cadavers, various reagents have been used. In recent years, commercially available embalming agents for cadaver restoration and preservation have been evaluated, but they have not been sufficiently compared. In this study, we successfully restored fingerprints from a highly dried, almost mummified, unidentified cadaver. Five methods were attempted to restore fingerprints: three used previously reported reagents and two used commercially available embalming solutions at room temperature. The fingers were observed grossly after immersion for $1,2,3,5$, and 7 days. When all the specimens were well restored, fingerprints were taken by the inked impression method and by an indirect method using silicone rubber. The results indicated that $\mathrm{Na}_{2} \mathrm{CO}_{3}$, and Sofner ${ }^{\circledR}$ were more effective to restore clear fingerprints. The other solutions failed to produce optimal tissue softening and swelling, and the dermal ridges were ill-defined. The conventional $\mathrm{Na}_{2} \mathrm{CO}_{3}$ and the newly tested Sofner ${ }^{\circledR}$ were useful in that they restored better fingerprints in a shorter duration. $\mathrm{Na}_{2} \mathrm{CO}_{3}$ has to be prepared before use, whereas Sofner ${ }^{\circledR}$ can be used by simply diluting with water.
\end{abstract}

Keywords: Restoration; Fingerprint; Mummified; Individual identification

\section{Introduction}

Several methods are used in forensic sciences for individual identification of cadavers, such as sex, age, physical characteristics, blood type, DNA test results, dental findings, and fingerprints. In particular, fingerprints are widely used as the most reliable means of personal identification, because no two persons have identical dermal ridge patterns, and the patterns of any one individual remain unchanged throughout life [1-4]. However, postmortem changes of the skin of fingers present problems with fingerprinting. Therefore, methods of taking fingerprints appropriate for the state of rigor mortis, putrefaction, mummified, and burned bodies were investigated [5]. To restore fingerprints of mummified cadavers, various reagents have been reported. In recent year, commercially available embalming agents for cadaver restoration and preservation have been evaluated [6,7], but they have not been compared sufficiently.

In this study, we compared three conventional reagents and two embalming solutions for restoring fingerprints of a highly dried, almost mummified, unidentified cadaver.

\section{Materials and Methods}

\section{Background}

A male cadaver was found in an empty house in winter. The body was in various stages of advanced decomposition and profusely infested by fly maggots. Large portions of the integument, including that of the hands and feet, had mummified. Autopsy was required by the police for diagnosing the cause of death. The results of our judicial autopsy indicated that the remains were those of a middle-aged male, estimated to have been dead for approximately half a year. The identity of the man was not known, and individual identification was needed. Investigation of the fingerprints was the first choice, because there was nothing that could be used to identify the individual. However, the cadaver had mummified, and the skin of all fingers was too dry and rigid to take the fingerprints. Therefore, restoration of fingerprints was required as the first step.

\section{Materials}

Both hands of the mummified cadaver were examined (Figure 1). After carefully cleaning the hands with physiological saline, definite injury was not observed in the right palm and six fingers: thumb, index finger, middle finger, ring finger, little finger of the right hand and little finger of the left hand. Because these fingers were likely to retain their dermal ridge patterns, we decided to use them in our attempt to examine the fingerprints.

\section{Methods}

Several methods have been reported for the restoration of fingerprints from different forensic caseworks. The methods used in this study were based on those published reports. Three restoration reagents were used: solution $\mathrm{A}$ was sodium hydroxide-glycerin $(5 \%$ $\mathrm{NaOH} 100 \mathrm{ml}$, glycerin $3 \mathrm{ml})$; solution $\mathrm{B}$ was sodium carbonate $\left(\mathrm{Na}_{2} \mathrm{CO}_{3}\right.$ $10 \mathrm{~g}$, 95\% ethanol $316 \mathrm{ml}$, distilled water $684 \mathrm{ml}$ ); and solution $\mathrm{C}$ was TIOAR-330 ${ }^{\circledR}$ (formalin 30\%, methanol 15\%; IMS Co.). Another two commercially available embalming agents; Sofner ${ }^{\circledR}$ (methanol 5.5\%, ethylene glycol 11\%, ethylene diamine tetraacetic acid 16\%; ESCO Co.) and Special Arterial Chemical- LITHOL Index $32^{\circledR}$ (formaldehyde $32 \%$, methanol 5.7\%, ethylene glycol 5.5\%; ESCO Co.), were also used to recreate the fingerprints.

The embalming solutions were diluted to $50 \%, 25 \%, 12.5 \%$ and $6 \%$ before use. The fingers were divided into various portions for testing

*Corresponding author: Jian Tie, Division of Legal Medicine,Department of Social Medicine, Nihon University School of Medicine, 30-1 Oyaguchi, Kamimachi, Itabashi-ku, Tokyo 173-8610, Japan, Tel: +81-3-3972-8111 ex. 2277; Fax: +81-33958-7776 ; E-mail: tetsu,ken@nihon-u.ac.jp

Received September 12, 2011; Accepted October 14, 2011; Published October 15, 2011

Citation: Iwakami E, Uchigasaki S, Tie J (2011) Restoration of Fingerprints from a Mummified Cadaver. J Forensic Res S6:001. doi:10.4172/2157-7145.S6-001

Copyright: (c) 2011 Iwakami E, et al. This is an open-access article distributed under the terms of the Creative Commons Attribution License, which permits unrestricted use, distribution, and reproduction in any medium, provided the original author and source are credited. 
Citation: Iwakami E, Uchigasaki S, Tie J (2011) Restoration of Fingerprints from a Mummified Cadaver. J Forensic Res S6:001. doi:10.4172/21577145.S6-001

different solutions at various concentrations as follows: distal and proximal phalanges of right thumb; distal and proximal thenar regions of right palm; distal, middle and proximal phalanges of right index finger; distal, middle and proximal phalanges of right middle finger; distal, middle and proximal phalanges of right ring finger; distal and middle phalanges of right little finger; and distal and middle phalanges of left little finger. Each phalanx was wrapped in gauze material soaked with each reagent at room temperature, and observed grossly after treatment for 1, 2, 3, 5 and 7 days. When all the specimens were well restored, fingerprints were taken by the inked impression method and by an indirect method using silicone rubber.

\section{Results}

The important precaution taken in recreating the fingerprints of this case was to allow the dried and rigid skin of the mummified fingers to hydrate and elevate sufficiently, and prevent injury to the skin of the fingers during the experimentation. The five restoration solutions yielded different results.

The finger portion treated with solution A softened rapidly one day later, although the dermal ridges remained ill-defined. After two days of treatment, the skin swelled, the tissue cleared, and the dermal ridges became well-defined. However, no clear fingerprints could be taken (Figure 2). The finger continued to imbibe and swell and, became gelatinous beyond recognition after treatment for 7 days.

The finger portion treated with solution B gradually changed from dark brown to flesh color, and softened 1 day later. The dermal ridges became well-defined two days later (Figure 3), and allowed the



Figure 1: Both hands of the mummified cadaver.

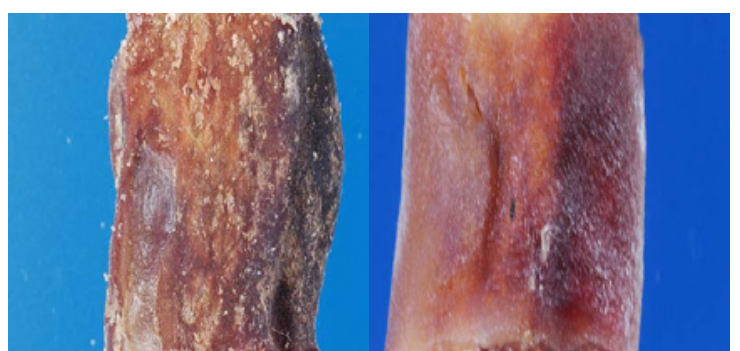

Figure 2: The media phalanx of right middle finger treated with solution $A$ for two days (right), compared with before treatment (left). The tissue had cleared, and the dermal ridges became well-defined. However, no clear fingerprints could be taken.



Figure 3: The distal phalanx of the right little finger treated with solution $B$ for two days (right) compared with before treatment (left). After treatment, the finger changed from dark brown to flesh color, softened, and the dermal ridges became well-defined.

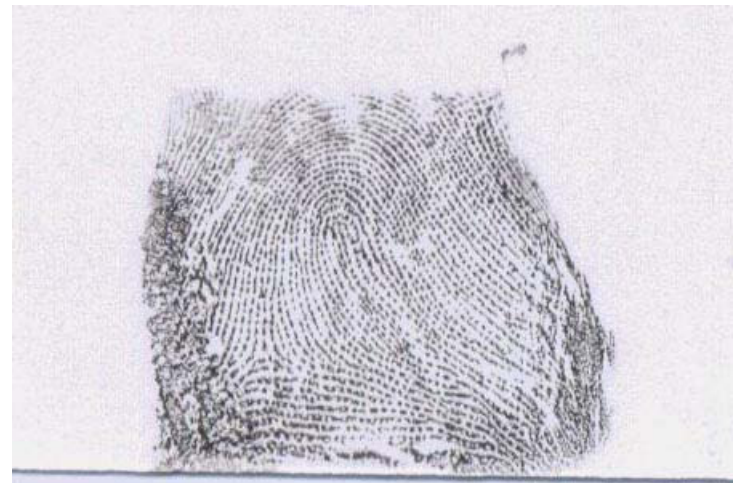

Figure 4: A fingerprint of the right little finger treated with solution $B$ for two days, taken by inked impression method.

impression of clear fingerprints (Figure 4). From five days onward, the finger continued to swell, and the skin grooves became shallow, making the dermal ridges ill-defined.

The finger portion treated with solution $\mathrm{C}$ became stained with the reagent (pink). Regardless of the dilution of the solution, tissue softening was slow. Seven days later, softening and swelling remained insufficient, and the dermal ridges were ill-defined (Figure 5).

The finger portion treated with Sofner ${ }^{\circledR}$ gradually became bleached. Regardless of the dilution of the solution, treatment produced well defined dermal ridges. At a concentration of $25 \%$ or lower, the phalanges softened properly three days later (Figure 6), and clear fingerprints were obtained (Figure 7). Thereafter, softening progressed slowly until day 7 .

The finger portion treated with LITHOL Index $32^{\circledR}$ became stained with the reagent (pink). Regardless of the dilution of the solution, treatment resulted in well defined dermal ridges. Softening of the skin became slower as the concentration of the reagent increased. Only the fingers treated with a $6 \%$ dilution of the reagent exhibited slight softening from 1 to 7 days (Figure 8), but clear fingerprints could not be obtained after treatment for 3 days.

Therefore, solutions B and Sofner ${ }^{\circledR}$ both produced good results in the restoration experiment. 
Citation: Iwakami E, Uchigasaki S, Tie J (2011) Restoration of Fingerprints from a Mummified Cadaver. J Forensic Res S6:001. doi:10.4172/21577145.S6-001

\section{Discussion}

A number of techniques to obtain fingerprints from mummified cadavers have been proposed in the literature. Although the reagents and procedures have been reported to produce good results, reagent preparation and the procedures are time-consuming. "Tanning solution" involves the removal of tissue from the glycerin-resistant phalanges, followed by the action of a $50 \%$ sulfuric acid in saturated saline solution on the specimens for 72 hours [8]. A second method uses a detergent solution containing disodium ethylenediamine tetracetic acid ( $\mathrm{pH}$ 7.5) [9], but the method requires reagent preparation and $\mathrm{pH}$ adjustment.

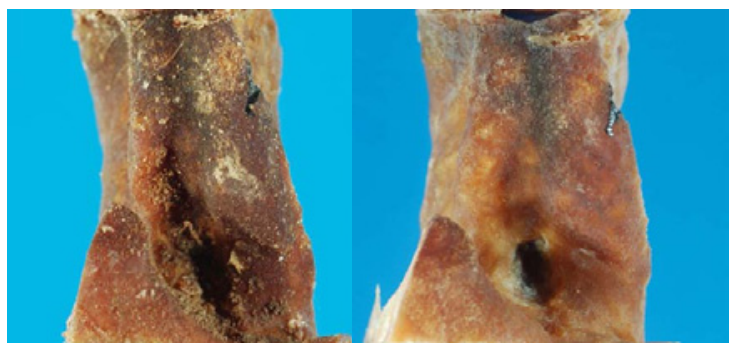

Figure 5: The proximal phalanx of the right middle finger treated with solution $C$ for seven days (right) compared with before treatment (left). The finger became softened, but swelling remained insufficient, and the dermal ridges were illdefined.

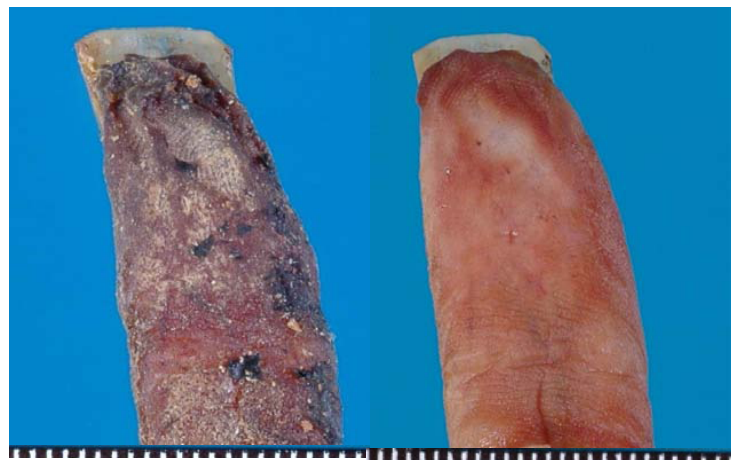

Figure 6: The distal phalanx of the left little finger treated with Sofner ${ }^{\circledR}$ at a concentration of $25 \%$ for three days (right) compared with before treatment (left). The finger became bleached, and the dermal ridges were well-defined.

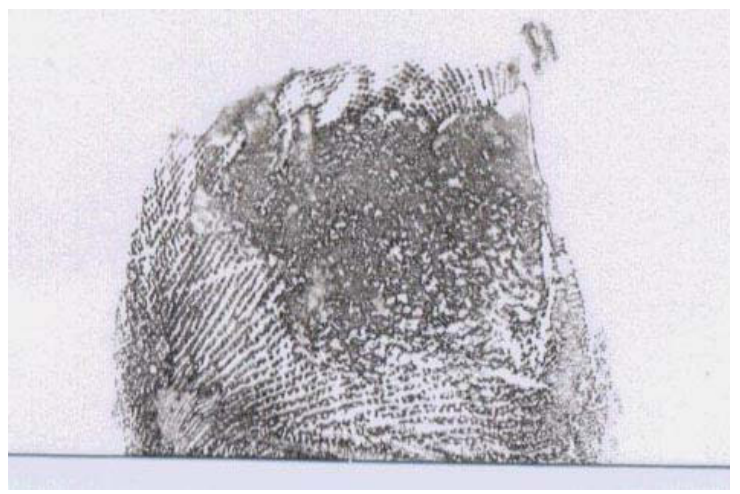

Figure 7: A fingerprint of the left little finger treated with Sofner ${ }^{\circledR}$ for two days taken by the inked impression method.

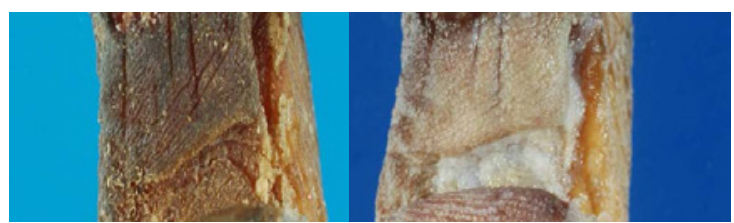

Figure 8: The proximal phalanx of the right little finger treated with LITHOL Index $32^{\circledR}$ for three days (right) compared with before treatment (left). The finger became stained with the reagent (pink), but no clear dermal ridges could be observed.

Alkaline solutions have been used relatively frequently. For example, the action of $1 \mathrm{~N}$ potassium hydroxide solution on skin specimens for 48 hours at $30^{\circ} \mathrm{C}$ results in epidermolysis, and the exposed dermis can be observed [10]. This method requires preparation of the reagent and temperature management during testing. Furthermore, it has been noted that an alkaline solution at a high concentration or temperature may destroy the dermis.

Another method uses $\mathrm{NaOH}$-glycerin, an alkaline solution that can be prepared and used relatively easily. A comparative experiment reported that $\mathrm{NaOH}$-glycerin was more effective than water, physiological saline, and antifoaming solution in restoring fingerprints [11]. Thus, $\mathrm{NaOH}$-glycerin was tested in this study (solution A), but softening and swelling progressed before dermal ridges became welldefined, and the specimen became gelatinous 2 days later, with swelling of the tissue. This was probably due to the excessive corrosive action of sodium hydroxide on the skin. Therefore, the use of an alkaline solution requires prior determination of the optimal concentration.

To avoid tissue destruction, $\mathrm{Na}_{2} \mathrm{CO}_{3}$ solution in $95 \%$ ethanol (solution B) was used. Treatment with solution B for 24 hours has been reported to soften skin specimen [12]. In this study, softening progressed favorably during treatment with solution $\mathrm{B}$, and clear fingerprints were obtained 2 days later. Since its corrosive action on the skin is not strong, it restores the ridges in the epidermis but does not affect the dermis, thereby facilitating the impression of fingerprints suitable for routine fingerprint investigation.

In recent years, the use of commercially available embalming solutions has been studied to eliminate the need for the complicated solution preparations and temperature management. Embalming is a procedure that repairs, preserves, disinfects, and maintains a dead body safely and cleanly for certain periods by replacing the blood with preservatives. Overnight immersion in a 1:1 mixture of Metaflow ${ }^{\circledR}$ and Restorative ${ }^{\circledR}$ has been reported to provide sufficient tissue softening for 20 hours, and subcutaneous injection of this solution allows impression of good fingerprints [6]. Metaflow ${ }^{\circledR}$ is a reagent for the drainage of blood, and Restorative ${ }^{\circledR}$ is a product for the repair and preservation of a dead body. Both reagents were manufactured by Dodge Chemical Company, Cambridge, Massachusetts. Later, methods using only reagents corresponding to Restorative ${ }^{\circledR}$ were studied. A comparison of FLESHTONE ${ }^{\circledR}$ (formalin 28\%, glutaraldehyde 14\%; IMS Co.) and TIOAR-330 ${ }^{\circledR}$ (solution C in the present study) reported that solution $\mathrm{C}$ softened and denuded the epidermis, and was useful to expose the dermis [7]. However, Metaflow ${ }^{\circledR}$ and Restorative ${ }^{\circledR}$ are currently not commercially available.

Thus, in this study, we used reagents equivalent to Metaflow ${ }^{\mathbb{B}}$ and Restorative ${ }^{\circledR}$ to test methods of restoring dermal ridges in a mummified cadaver. From the commercially available embalming 
Citation: Iwakami E, Uchigasaki S, Tie J (2011) Restoration of Fingerprints from a Mummified Cadaver. J Forensic Res S6:001. doi:10.4172/21577145.S6-001

solutions, Sofner ${ }^{\circledR}$ and Special Arterial Chemical- LITHOL Index32 ${ }^{\circledR}$ were selected as equivalent reagents for Metaflow ${ }^{\circledR}$ and Restorative ${ }^{\circledR}$, respectively. Since both solutions are used for embalming in undefined concentrations, the optimal concentrations for use were not known. Therefore, we used serial dilutions of each solution. While LITHOL Index $32^{\circledR}$ was expected to be suitable for the restoration of dermal ridges, our results showed that Sofner ${ }^{\circledR}$ was more useful. In particular, LITHOL Index $32^{\circledR}$ at a concentration of $25 \%$ or higher did not cause tissue softening, probably due to the potent fixative effect of formalin.

To find a useful method for restoring the dermal ridges (fingerprints) of a mummified cadaver, five reagents were tested in this study. Not all methods that have been reported to give good results produced useful changes during 7 days of observation. This was presumably due to the degree of dryness and the shape of the specimens used. Therefore, it is desirable to perform a preliminary study to determine the optimal concentration and duration of action of the reagents before application to the specimen.

In this study, the conventional solution B and the newly tested Sofner ${ }^{\circledR}$ were considered more effective in that they restored better fingerprints in shorter durations than the others. Solution B must be prepared before use, whereas Sofner ${ }^{\circledR}$ can be used conveniently by simply diluting with water. Commercially available products such as solution $\mathrm{C}$ may be removed from the market at any time. Moreover, their formulae are not known, making it impossible to prepare these reagents in the laboratory. On the other hand, the composition of solution B is known. Thus, solution B is superior in that it can be prepared easily as long as the two ingredients are available.

Fingerprints were taken by the inked impression method or an indirect method using silicone rubber. If the dermal ridges are restored not on fingertips, but after separation of the skin from the bone, special technique is needed to impress fingerprints [9]. For this purpose, a method using $\mathrm{T}$ pins has been developed [8]. Since the reported restoration method using an embalming solution exposes the dermis, the dermal ridges are stained with $0.05 \%$ toluidine blue solution for observation [13]. When a specimen is taken with the epidermis partially sloughing and the dermis exposed, a single dermal ridge may appear as a double fine line, requiring particularly careful handling. However, the present methods allowed us to restore the dermal ridge patterns and obtain clear fingerprints easily by the inked impression method. In this respect also, it is an excellent method of fingerprint restoration. Finally, the police checked the fingerprints in their files, and successfully identified the cadaver.

\section{Acknowledgments}

We thank the Department of Legal Medicine, Kyorin University School of Medicine and Sunseal Co., Ltd. for providing three valuable embalming solutions. We are also grateful to Mr. Yoshihiro Mitsuki and Mr. Takayuki Ota and Mr. Nobuo Onuki, Criminal Investigation Laboratory, Saitama Prefectural Police Headquarters for their cooperation in the taking of fingerprints

\section{References}

1. Caplan RM (1990) How fingerprints came into use for personal identification. J Am Acad Dermatol 1: 109-114.

2. Stigler SM. (1995) Galton and identification by fingerprints. Genetics 3: 857860 .

3. Garruto RM, Plato CC (1991) Fingerprints, palms, and soles: historica transitions. Birth Defects Orig Artic Ser 2: 7-18.

4. Walker R, Parsche F, Bierbrier M, McKerrow JH (1987) Tissue identification and histologic study of six lung specimens from Egyptian mummies. Am J Phys Anthropol 72: 43-48.

5. Kahana T, Grande A, Tancredi DM, Penalver J, Hiss J (2001) Fingerprinting the deceased: traditional and new techniques. J Forensic Sci 4: 908-912.

6. Haglund WD. (1988) A technique to enhance fingerprinting of mummified fingers. J Forensic Sci 5: 1244-1248.

7. Watanabe T, Shoji T, Takagi T, Kagehara B, Kajiwara M et al.(1995) A method of fingerprint collection from high degree parched fingers. Kyorin igaku zasshi 26: 467-472.

8. Richardson L, Kade H (1972) Readable fingerprints from mummified or putrefied specimens. J Forensic 2: 325-328.

9. Zugibe FT, Costello JT (1986) A new method for softening mummified fingers J Forensic Sci 32: 726-731.

10. Okajima M (1984) Dermatoglyphic examination of the dermal surface of bodies in advanced postmortem condition. Forensic Sci Int 3: 209-218.

11. Ishibashi H, Fujita M, Yamaguchi K, Shoji S (1977) Legal medicine studies on the mummification cadaver (author's translation). Hokkaido igaku zasshi 6 337-339.

12. Schmidt CW, Nawrocki SP, Williamson MA, Marlin DC (2000) Obtaining fingerprints from mummified fingers: a method for tissue rehydration adapted from the archeological literature. J Forensic Sci 4: 874-875.

13. Okajima M (1988) Supplementary review of dermatoglyphic researches. Res pract forens med 31: 1-12. 